\title{
Vertebral Deformities in Female Patients with Osteoporosis: Influence of Trauma and Bone Mineral Density
}

\author{
KATSUYA KANESAKI \\ Department of Orthopaedic Surgery, Kurume University School of Medicine, \\ Kurume 830-0011, Japan
}

\begin{abstract}
Summary: Aging societies have an increased incidence of fractures caused by osteoporosis. Vertebral compression fractures occur most frequently in the thoracolumbar vertebrae of elderly individuals. The severe pain caused by this type of fracture causes many patients to become bedridden. However, the initial pain generally diminishes after around 1 month and is replaced by dull pain in the lumbar and lumbosacral regions. We carried out a cross-sectional and longitudinal study of these fractures in 328 female outpatients with osteoporosis. All subjects initially presented at our hospital with back pain and bone mineral density measurements were taken using both dual energy X-ray absorptiometry and quantitative computed tomography. Our main findings were as follows. Decreased BMD appears to be one cause of vertebral body deformities. However, we found it difficult to predict whether deformities will progress or cause additional deformities of adjacent vertebral bodies, because the key contributing factors include not only BMD and age but also lifestyle and activity patterns. In addition, none of the drug administration methods investigated here resulted in a notable increase in BMD.
\end{abstract}

Key words osteoporosis, vertebral fracture, bone mineral density, trauma

\section{INTRODUCTION}

Aging societies have an increased incidence of fractures caused by osteoporosis. Vertebral compression fractures in particular occur more frequently among the elderly and can result in patients becoming bedridden owing to severe back pain. Such compression fractures often occur in the thoracolumbar vertebrae, causing severe pain at the fracture site in the dorsolumbar area during the acute stage. This pain usually diminishes after about 1 month as the symptoms progress to the chronic stage. At this stage, spinal deformity caused by the vertebral compression fracture produces abnormal stress to the spinal support system, resulting in dull pain in the lumbar and lumbosacral regions.

Previous reports of vertebral compression frac- tures have tended to be cross-sectional studies and have not considered trauma. Such studies have shown that the threshold of fracture occurrence varies with bone mineral density (BMD). However, few longitudinal studies have been performed to confirm these results. Here we report on a long-term study of BMD in outpatients with osteoporosis in which used dual energy X-ray absorptiometry (DEXA) and quantitative computed tomography (QCT) were used to investigate the relationship between vertebral body deformities, trauma and BMD.

\section{SUBJECTS AND METHODS}

The subjects of this study were 328 female patients with osteoporosis who presented at our hospital with back pain between December 1988 and

Received for publication June 8, 2004

Editorial correspondence: Katsuya Kanesaki, M.D., Department of Orthopaedic Surgery, Kurume University School of Medicine, 67 Asahimachi, Kurume 830-0011, Japan. Tel: +81-942-35-3311 Fax: +81-942-39-0709 E-mail: kintaro@mojih.rofuku.go.jp

Abbreviations: BMD, bone mineral density; DEXA, dual energy X-ray absorptiometry; DPA, dual photon absorptiometry; QCT, quantitative computed tomography. 
August 1995. BMD measurements were taken in all subjects using both DEXA and QCT at the initial examination. The subjects ranged in age from 50 to 91 years (mean age $=67$ years). Follow-up observations were carried out on 105 patients from this initial group for a period ranging from 6 to 69 months (mean follow up period $=16$ months). Plane radiographs showing lateral views of the 10th thoracic vertebra through to the 5th lumbar vertebra were taken in all subjects at the time of the initial BMD measurements. These were used to classify the patients into five groups according to the type of vertebral deformity present: no deformity, irregular endplate, wedged vertebra, flat vertebra or fish vertebra. The relationship between the number of deformities, BMD and history of trauma was examined. The distal part of the radius and the lateral part of the 3rd lumbar vertebra were measured using DEXA, and the 3rd lumbar vertebra was measured using QCT. Measurements were taken using a Hologic QDR1000W (DEXA), a TCT-70A (CT) (Toshiba Corp.) and a B-MAS Bone Mass Phantom (Chugai Pharmaceutical Co., Ltd.). All results were statistically analyzed using Fisher computer software.

\section{RESULTS}

The correlation coefficients (R) for the relationship between age and BMD were as follows: -0.1529 for the side of the 3rd lumber vertebra, -0.5940 for the radius total, -0.5516 for the radius
$1 / 3,-0.5849$ for the radius MID, -0.3854 for the radius UD and -0.5300 for the QCT. The BMD of the 3rd lumbar vertebra showed the lowest correlation with age and that of the radius total showed the highest correlation. Therefore, the radius total DEXA measurement of BMD was used in the following examinations and analyses because it had the highest correlation with age.

The mean BMD values for each age group determined using DEXA were as follows: $0.424 \pm 0.05$ $\mathrm{g} / \mathrm{cm}^{2}$ for the group aged 50-59 years; $0.391 \pm 0.05$ $\mathrm{g} / \mathrm{cm}^{2}$ for the group aged $60-69$ years; $0.347 \pm 0.05$ $\mathrm{g} / \mathrm{cm}^{2}$ for the group aged 70-79 years; and 0.304士 $0.04 \mathrm{~g} / \mathrm{cm}^{2}$ for the group aged $\geq 80$ years. There were significant differences in mean BMD between the different age groups.

The mean BMD values obtained using QCT were as follows: $98.15 \pm 34.3 \mathrm{mg} / \mathrm{cm}^{3}$ for the group aged $50-59$ years; $71.57 \pm 35.90 \mathrm{mg} / \mathrm{cm}^{3}$ for the group aged $60-69$ years; $48.91 \pm 38.17 \mathrm{mg} / \mathrm{cm}^{3}$ for the group aged $70-79$ years; and $31.50 \pm 31.50 \mathrm{mg} / \mathrm{cm}^{3}$ for the group aged $\geq 80$ years. As with the DEXA values, there were significant differences in mean BMD between the different age groups (Fig. 1).

The numbers of patients with vertebral body deformities in the different age groups were as follows. Within the group aged 50-59 years, 60 patients (87\%) had no deformity, nine patients (13\%) had a single vertebral deformity and no patients had deformities involving multiple vertebral bodies. Within the group aged 60-69 years, 98 patients (75.4\%) had

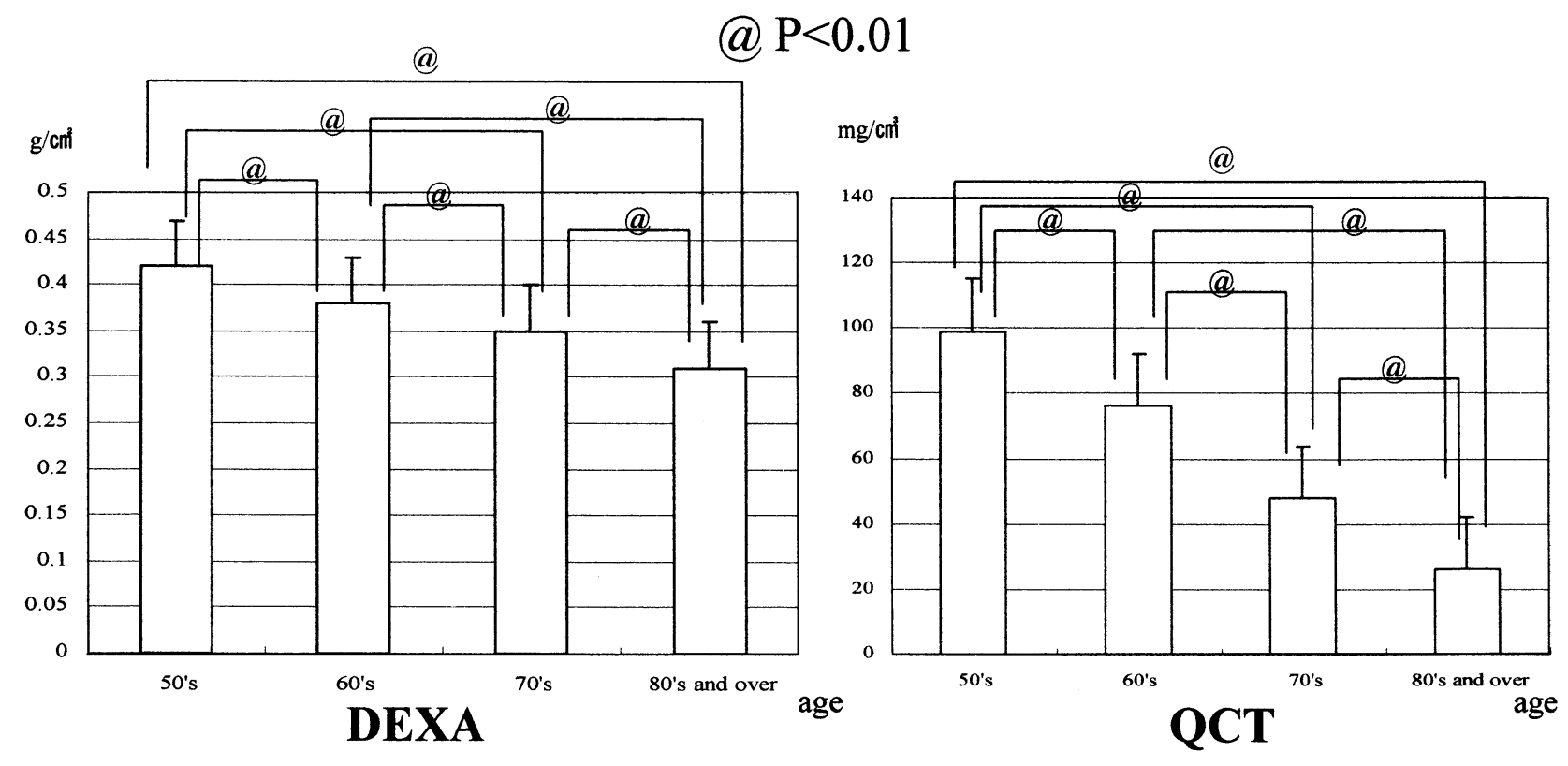

Fig. 1. There were significant differences in mean BMD between the different age groups in both DEXA and QCT. 
no deformity, 25 patients (19.2\%) had a single vertebral deformity and seven patients $(5.4 \%)$ had deformities involving multiple vertebral bodies. Within the group aged $70-79$ years, 60 patients $(54.5 \%)$ had no deformity, 32 patients $(29.1 \%)$ had a single vertebral deformity and 18 patients $(16.4 \%)$ had deformities involving multiple vertebral bodies. In the group aged $\geq 80$ years, six patients $(31.6 \%)$ had no deformity, five patients $(26.3 \%)$ had a single vertebral deformity and eight patients $(42.1 \%)$ had deformities involving multiple vertebral bodies. These data revealed a significant trend: the higher the age, the higher the rate of fracture and the greater the number of deformed vertebral bodies ( $p<0.01$ ) (Fig. 2).

The relationships between the different types of vertebral body deformity and age were investigated. The mean ages for each group were as follows: 65.2 \pm 7.8 years for the 224 patients with no deformities; $69.2 \pm 6.4$ years for the 16 patients with irregular endplates; $68.3 \pm 7.3$ years for the 51 patients with wedged vertebrae; $75.5 \pm 8.9$ years for the 22 patients with flat vertebrae; and $76.7 \pm 7.2$ years for the 15 patients with fish vertebrae. There was a significant difference between the mean age of those patients who had no deformities and those with flat vertebrae or fish vertebrae $(p<0.01)$. There was also a signifi- cant difference between the mean ages of patients with irregular endplates or wedged vertebrae and those with fish vertebrae $(\mathrm{p}<0.05)$ (Fig. 3).

Subjects were classified into three groups according to the degree of trauma. The first group contained individuals who had no trauma prior to the onset of symptoms (the no trauma group). The second group comprised individuals whose symptoms had occurred as a result of slight injury caused by everyday activities, such as lifting a heavy object or a fit of coughing (the slight injury group.) The third group consisted of individuals whose symptoms had occurred as a result of significant trauma, such as a fall (the noticeable trauma group).

The relationship between the number of vertebral deformities and BMD was investigated among the 258 members of the no trauma group: the 204 patients with no deformity had mean BMD values of $0.395 \pm 0.06 \mathrm{~g} / \mathrm{cm}^{2}$ (DEXA) and $80.5 \pm 38.2 \mathrm{mg} / \mathrm{cm}^{3}$ (QCT); the 33 patients with a single vertebral deformity had mean BMD values of $0.360 \pm 0.06 \mathrm{~g} / \mathrm{cm}^{2}$ (DEXA) and $55.6 \pm 34.9 \mathrm{mg} / \mathrm{cm}^{3}$ (QCT); and the 21 patients with multiple vertebral deformities had mean BMD values of $0.304 \pm 0.05 \mathrm{~g} / \mathrm{cm}^{2}$ (DEXA) and $24.2 \pm 45.2 \mathrm{mg} / \mathrm{cm}^{3}$ (QCT). There were significant differences between the BMD values within the no

$\square$ none $\square$ one $\mathbb{\mathbb { N }}$ two or more

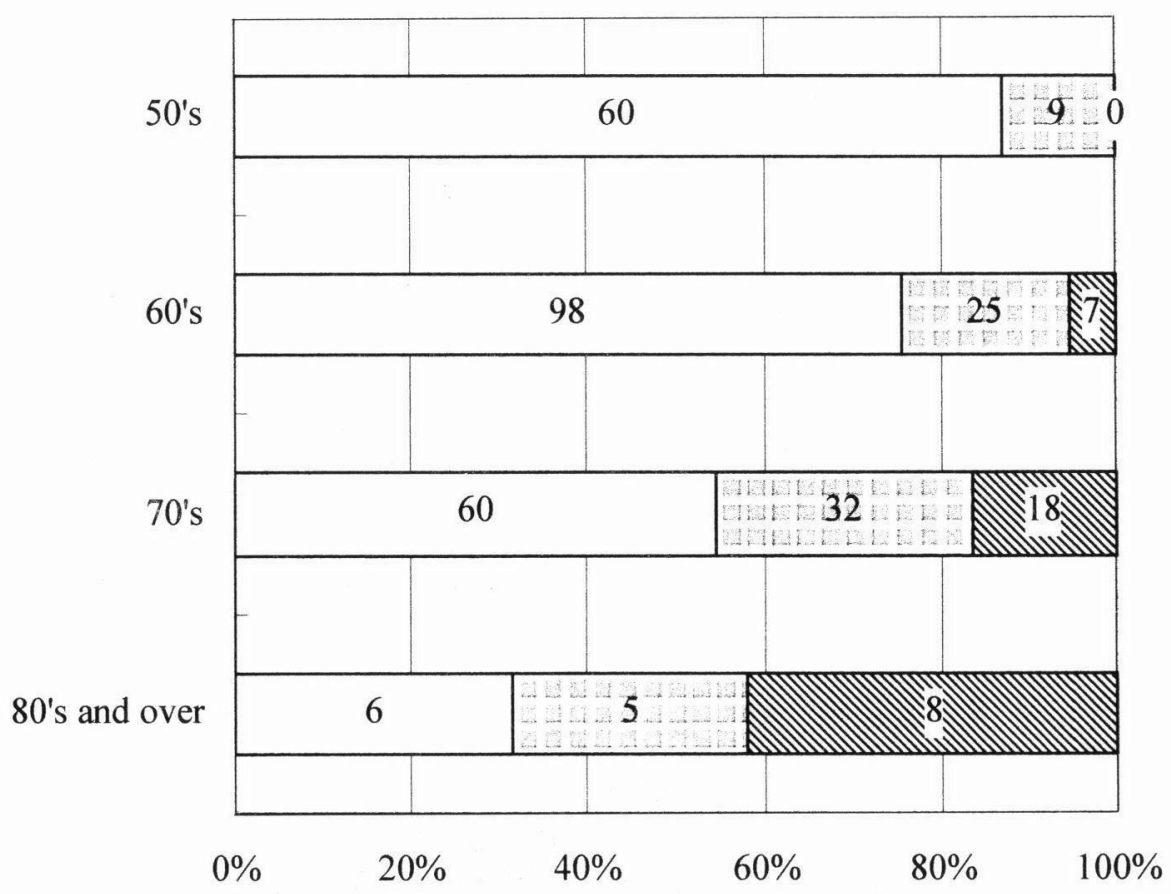

Fig. 2. There was a significant trend indicating that fracture rates and the number of deformed vertebral bodies increased with age. 


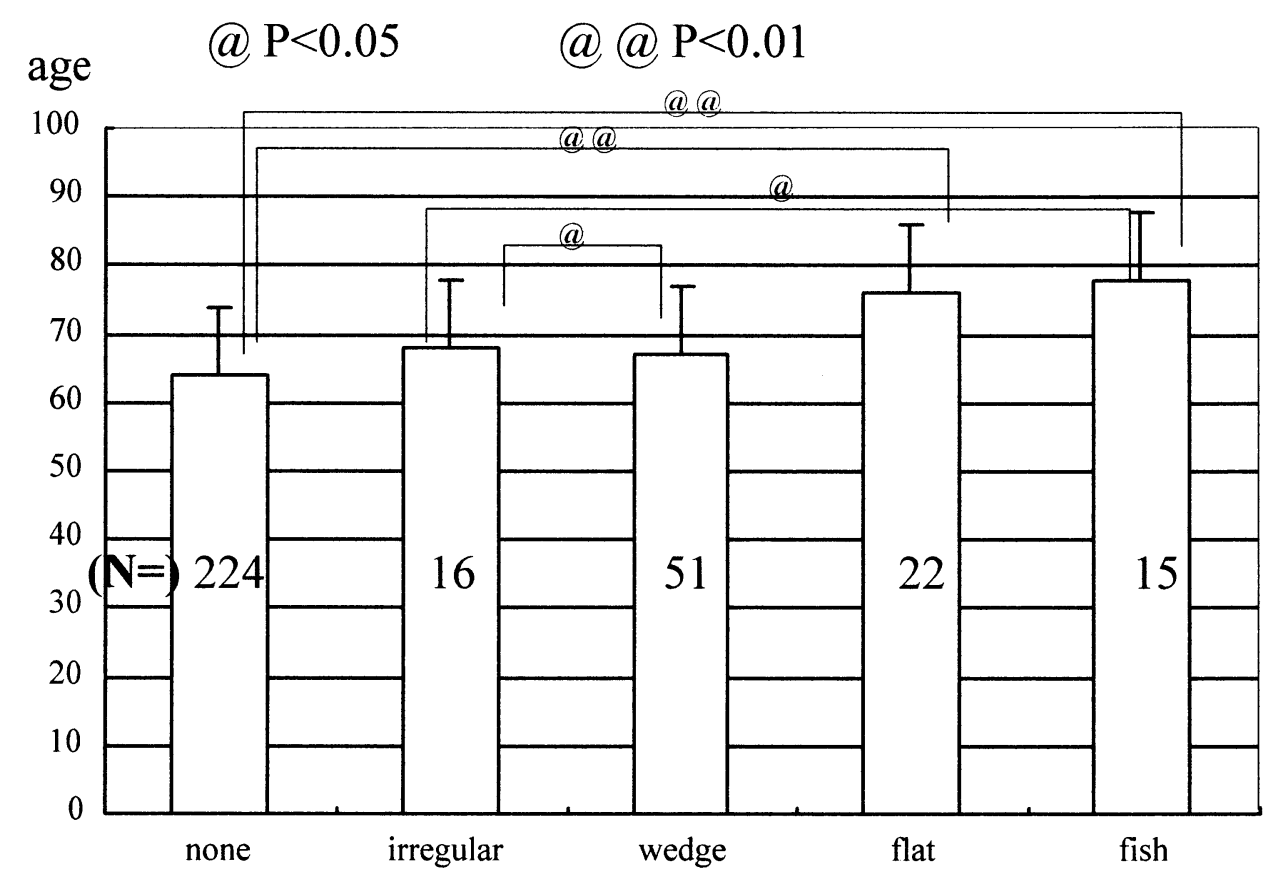

Fig. 3. There was a significant difference between the mean ages of the patients in each of the deformity categories.

@ $\mathrm{P}<0.01$

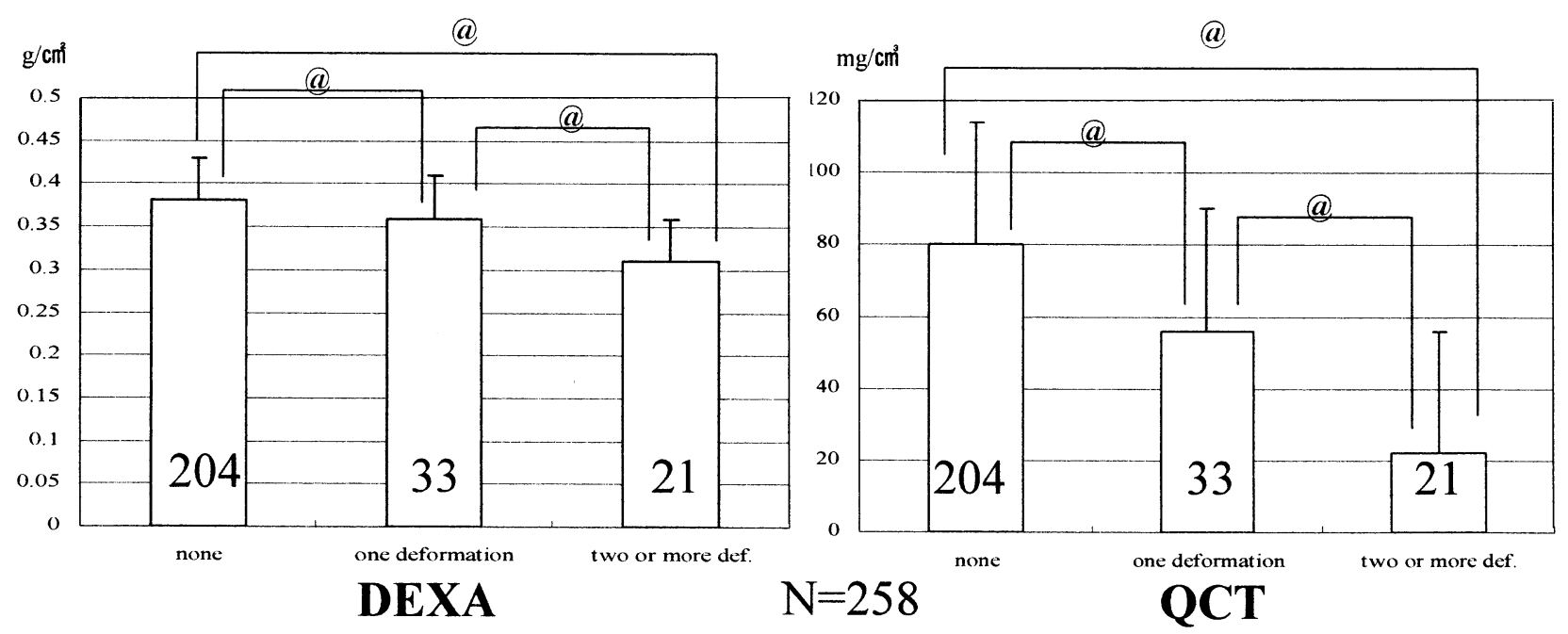

Fig. 4. There were significant differences between the BMD values within the no trauma group according to number of deformities.

trauma group according to number of deformities $(\mathrm{p}<0.01)$ (Fig. 4).

The mean BMD values of the 70 patients reporting trauma, including both the light trauma and noticeable trauma groups, were as follows: the 20 patients with no deformity had mean BMD values of $0.372 \pm 0.06 \mathrm{~g} / \mathrm{cm}^{2}$ (DEXA) and $70.37 \pm 36.5 \mathrm{mg} / \mathrm{cm}^{3}$ (QCT); the 38 patients with a single vertebral deformity had mean BMD values of $0.368 \pm 0.05 \mathrm{~g} / \mathrm{cm}^{2}$ (DEXA) and $44.4 \pm 37.1 \mathrm{mg} / \mathrm{cm}^{3}$ (QCT); and the 12 
patients with multiple vertebral deformities had mean BMD values of $0.318 \pm 0.05 \mathrm{~g} / \mathrm{cm}^{2}$ (DEXA) and $20.7 \pm 17.7 \mathrm{mg} / \mathrm{cm}^{3}$ (QCT). There were no significant differences between the number of deformities and the mean BMD within this group.

In order to determine the rate of occurrence of vertebral deformities associated with different BMD values, the DEXA measurements were classified into eight groups separated by intervals of $0.05 \mathrm{~g} / \mathrm{cm}^{2}$. The rate of incidence of deformities was first examined among the 258 patients in the no trauma group. Deformities were observed at the following frequencies: $100 \%$ of patients with a BMD $<0.25 \mathrm{~g} / \mathrm{cm}^{2}$; $60.8 \%$ of patients with a BMD of $0.25-0.30 \mathrm{~g} / \mathrm{cm}^{2}$; $33.2 \%$ of patients with a BMD of $0.30-0.35 \mathrm{~g} / \mathrm{cm}^{2}$; $11.9 \%$ of patients with a BMD of $0.35-0.40 \mathrm{~g} / \mathrm{cm}^{2}$; $10.7 \%$ of patients with a BMD of $0.40-0.45 \mathrm{~g} / \mathrm{cm}^{2}$; $12.4 \%$ of patients with a BMD of $0.45-0.50 \mathrm{~g} / \mathrm{cm}^{2}$; and $0 \%$ of patients with a BMD $\geq 0.50 \mathrm{~g} / \mathrm{cm}^{2}$. By contrast, among the patients within the trauma groups, four of the five patients $(80 \%)$ with a BMD $\geq 0.45 \mathrm{~g} / \mathrm{cm}^{2}$ had a single vertebral body deformity. There was no significant difference between the groups.

The QCT values were classified into seven groups separated by intervals of $20 \mathrm{mg} / \mathrm{cm}^{3}$. Vertebral deformities were observed at the following frequencies: $66.7 \%$ in patients with a BMD $<20 \mathrm{mg} / \mathrm{cm}^{3} ; 28.4 \%$ in patients with a BMD of $20-40 \mathrm{mg} / \mathrm{cm}^{3} ; 17 \%$ in patients with a BMD of $40-60 \mathrm{mg} / \mathrm{cm}^{3} ; 22 \%$ in patients with a BMD of $60-80 \mathrm{mg} / \mathrm{cm}^{3} ; 12.5 \%$ in patients with a BMD of $80-100 \mathrm{mg} / \mathrm{cm}^{3} ; 4.2 \%$ in patients with a BMD of $100-120 \mathrm{mg} / \mathrm{cm}^{3}$; and $5 \%$ in patients with a BMD $\geq 120 \mathrm{mg} / \mathrm{cm}^{3}$. By contrast, among the patients within the trauma groups, deformities were present in $>80 \%$ of those with a BMD $\geq 80 \mathrm{mg} / \mathrm{cm}^{3}$. There was no significant difference between the groups.

Next, the relationship between the different types of vertebral deformity and trauma was examined. A total of 104 patients ( $37.1 \%$ of all subjects) had some kind of vertebral deformity, and 49 of these 104 patients $(47.1 \%)$ reported trauma: 18 of these patients (8\% of all subjects) had experienced slight trauma and 31 patients (13.1\% of all subjects) had experienced noticeable trauma. Among those with an irregular endplate, six cases $(37.5 \%)$ were caused by slight trauma and two cases $(12.5 \%)$ were caused by noticeable trauma. Among those with a wedged vertebra, the figures were seven cases (13\%) and 19 cases $(37.3 \%)$, respectively. Among those with a flat vertebra, the figures were three cases (13.7\%) and six cases (27.3\%), respectively. Among those with a fish vertebra, the figures were two cases $(13.3 \%)$ and four cases $(26.7 \%)$, respectively. There was no significant relationship between the type of vertebral deformity and trauma (Fig. 5).

In this longitudinal study, we followed up 105 of the patients for $>6$ months. During this period, the following results were observed: 60 patients $(57.1 \%)$ showed no occurrence of a vertebral deformity; 26 patients $(24.8 \%)$ showed no progression of an existing deformity; 10 patients $(9.5 \%)$ showed progression of an existing deformity; seven patients (6.7\%) developed a new deformity at a different vertebral body from an existing deformity; and only two patients (1.9\%) who had no previous deformity developed a new one. The 19 patients who showed a change in vertebral deformity during the follow-up period had either continuous clinical symptoms or developed new back pain.

The histories of trauma in these patients at the time of their first medical examination were as follows: 58 of the 60 patients $(96.7 \%)$ with no vertebral deformity had no history of trauma, six of the 10 patients $(60 \%)$ showing progression of an existing deformity had a history of noticeable trauma, and six of the seven patients (87.5\%) showing an additional deformity at a different vertebral body had no history of trauma (Fig. 6). The mean BMDs of these three groups at the time of the first medical examination were $0.386 \pm 0.05 \mathrm{~g} / \mathrm{cm}^{2}, 0.352 \pm 0.07 \mathrm{~g} / \mathrm{cm}^{2}$ and $0.332 \pm 0.04 \mathrm{~g} / \mathrm{cm}^{2}$ (DEXA), and $76.3 \pm 33.6 \mathrm{mg} / \mathrm{cm}^{3}$, $36.1 \pm 36.1 \mathrm{mg} / \mathrm{cm}^{3}$ and $50.3 \pm 29.8 \mathrm{mg} / \mathrm{cm}^{3}$ (QCT), respectively. There was no significant difference between the groups.

The correlation between these follow-up results and the vertebral deformities recorded during the initial medical examinations were as follows. Among the 25 patients who had no progression of an existing deformity during the follow-up period, 12 patients $(48 \%)$ had a wedged deformity at the time of the first medical examination, seven patients $(28 \%)$ had an irregular endplate and four patients $(16 \%)$ had a flat vertebra. Among the 10 patients who had progression of an existing deformity, two patients (20\%) had an irregular endplate, four patients (40\%) had a wedged deformity, and four patients (40\%) had a flat vertebra at the time of the first medical examination. The three patients who had a fish vertebral deformity at the time of the first medical examination showed no change in their deformity during the follow-up period.

To examine the relationship between changes in 
BMD and in vertebral deformities, we calculated the annual rate of change of BMD as follows. The BMD at the time of the initial medical examination was subtracted from the BMD measured during the follow-up period. This value was then divided by the BMD at the time of the initial medical examination.

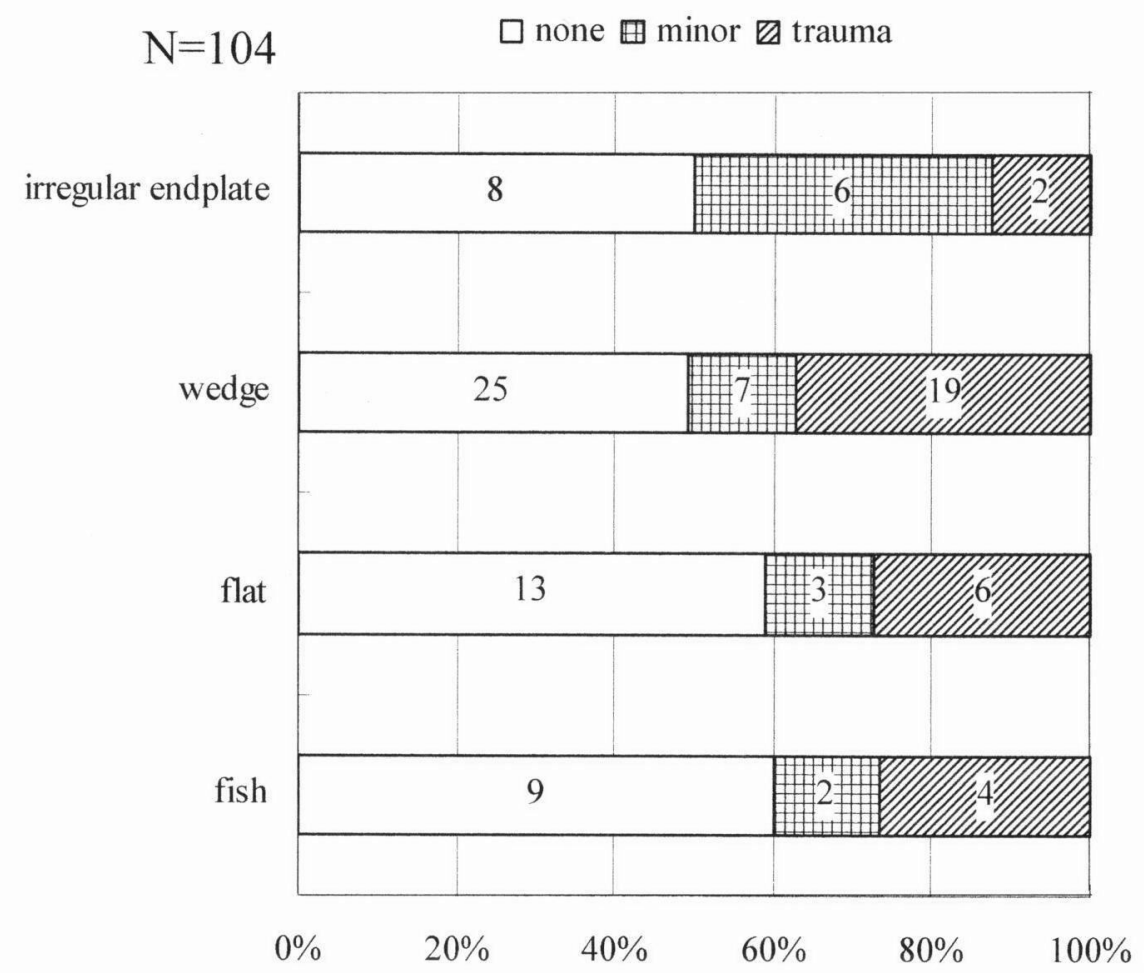

Fig. 5. There was no significant relationship between the type of vertebral deformity and trauma.

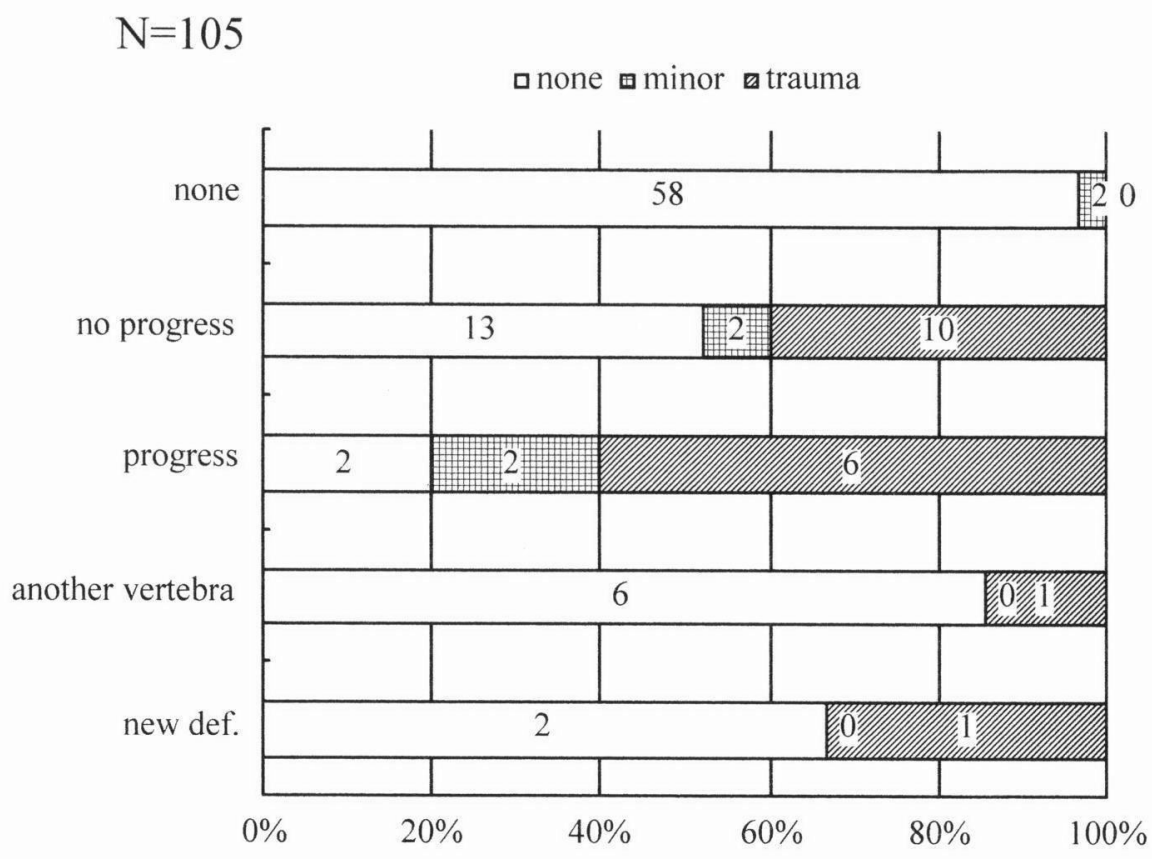

Fig. 6. The histories of trauma in patients at the time of the first medical examination. 
Finally, the result was converted into the rate of change of BMD per year.

Next, the subjects were classified into three groups according to the number of deformities present at the time of the initial medical examination: no deformity, a single vertebral deformity or multiple vertebral deformities. The relationship between the number of deformities and the rate of change of BMD was then investigated. According to the DEXA results, the rates of change of BMD in the three groups were as follows: $-0.0013 \pm 0.028$ in the group with no deformities, $-0.0025 \pm 0.042$ in the group with single vertebral deformities, and $-0.0085 \pm 0.043$ in the group with multiple vertebral body deformities. There was no significant difference between the three groups. According to the QCT results, rates of change of BMD in the three groups was $-0.035 \pm 0.264,-0.307 \pm 0.848$ and $-5.448 \pm 15.739$, respectively. Again, there was no significant difference between the three groups.

We then examined the relationship between the progression of deformities and the rate of change of BMD. According to the DEXA results, the rates of change of BMD were as follows: $-0.0013 \pm 0.027$ in the group who showed no deformities during the follow-up period, $0.0068 \pm 0.07$ in the group who showed no progression of existing deformities, $0.0095 \pm 0.041$ in the group who showed some progression of existing deformities, $-0.0285 \pm 0.052$ in the group who developed an additional deformity, and $-0.0392 \pm 0.048$ in the group who developed a deformity for the first time during the follow-up period. There was no significant difference between the groups. According to the QCT results, the rates of change were $-0.0351 \pm 0.264,-0.1352 \pm 0.796$, $-8.3038 \pm 19.407,-0.8350 \pm 0.784$ and $-0.2667 \pm$ 0.32 , respectively. The QCT results were similar to those produced by the DEXA, and there was no significant difference between the groups.

We next examined the relationship between the rate of change of BMD and the existence of vertebral deformities. The subjects were classified into two groups: one containing all individuals who showed no deformities during the follow-up period and the other containing all individuals who showed any kind of deformity during the follow-up period. According to the DEXA results, the group with no deformities (60 patients) had a rate of change of BMD of $-0.0012 \pm 0.028$ and the group with any kind of deformity (45 patients) had a rate of change of BMD of $-0.0043 \pm 0.0042$. According to the QCT results, these two groups had rates of change of BMD of $-0.035 \pm 0.2$ and $-1.981 \pm 9.12$, respectively. There were no significant differences between the two groups according to either the DEXA or the QCT results.

The relationship between the rate of change of BMD and change in vertebral deformities was then examined. Subjects were classified into two groups: one consisting of those individuals who had no deformity and those who showed no progression of an existing deformity (the group with no change), and the other consisting of all those who showed any change in deformity during the follow-up period (the group with change). According to the DEXA results, the group with no change in deformity (86 patients) had a rate of change of BMD of $-0.0012 \pm 0.031$, and the group with change in deformity (19 patients) had a rate of change of BMD of $-0.0196 \pm 0.045$. According to the QCT results, the two groups had rates of change of BMD of $-0.064 \pm 0.479$ and $-4.313 \pm 13.52$, respectively. There was no significant difference between the two groups according to the DEXA results; however, there was a significant difference according to the QCT results at the 5\% probability level $(\mathrm{p}<0.005)$. We therefore found that a decrease of BMD was one potential cause of progression in vertebral deformities (Table 1). In addition, 17 of the 19 patients $(89.5 \%)$ in the group with change in deformity showed a progression of their deformity without any noticeable trauma, such as falls, at least during the follow-up period. Only two of the 19 patients $(10.5 \%)$ reported a trauma that subsequently led to the progression of their deformity in

TABLE 1

The significant difference of the rate of change in QCT between the group with no progression (no change group) and that with progression (change group) indicated that a decrease of $B M D$ was one potential cause of progression in vertebral deformities

$\left.\begin{array}{lccr}\hline & \text { Number } & \text { Rate of change DEXA } & \text { Rate of change QCT } \\ \hline \text { group of no change } & 86 & 0.0012 \pm 0.013 & -0.064 \pm 0.479 \square @ @ @ ~ \\ \text { group of change } & 19 & -0.019 \pm 0.045\end{array}\right]$

@ N.S@@ 
subsequent radiographs.

Analysis of the relationship between change in vertebral deformities and history of trauma at the time of the initial medical examination showed that $60 \%$ of those individuals who showed progression of an existing deformity during the follow-up period had reported noticeable trauma at the initial medical examination. A total of $85.7 \%$ of those individuals who developed an additional deformity during the follow-up period reported no history of trauma at the initial medical examination. The rate of change of BMD was also examined in these two groups. According to the DEXA results, those who showed progression of an existing deformity had a rate of change of BMD of $-0.0089 \pm 0.048$, whereas those who developed an additional deformity had a rate of change of BMD of $-0.0378 \pm 0.048$. According to the QCT results, the rate of change of BMD in the two groups was $-12.14 \pm 26.01$ and $-0.979 \pm 0.853$, respectively. There was no significant difference between the two groups; in other words, BMD was not related to whether patients showed progression of an existing deformity or produced an additional deformity (Table 2).

Finally, we investigated the effects of the administration of bone-metabolism-related drugs, excluding non-steroidal anti-inflammatory agents, on changes in BMD during the follow-up period. We examined the influence of the internal use of active vitamin D3 and calcium preparations, ipriflavone and an intramuscular injection of calcitonin, both singly and in combination. Although the use of drugs is often determined by factors such as BMD and the number of vertebral body deformities at an initial medical examination, in this study the drugs were distributed randomly among the subjects. However, administration of the drugs was slightly biased according to BMD at the time of the initial medical examination: patients who received no drugs had BMDs of $0.375 \pm 0.06 \mathrm{~g} / \mathrm{cm}^{2}$ (DEXA) and 76.5 \pm 34.0 $\mathrm{mg} / \mathrm{cm}^{3}$ (QCT), whereas those who received a combination of active vitamin D3 and calcium preparations had initial BMDs of $0.391 \pm 0.03 \mathrm{~g} / \mathrm{cm}^{2}$ (DEXA) and $71.8 \pm 28.3 \mathrm{mg} / \mathrm{cm}^{3}$ (QCT). In addition, those who received no drugs had lower BMDs according to the DEXA results. The details of the administration of drugs according to the number of vertebral body deformities were as follows: 12 of the 17 patients (70\%) who received no drugs showed no deformities at the initial medical examination, and only one of the 14 patients $(7.0 \%)$ who showed multiple vertebral deformities at the initial medical examination did not receive drugs. However, no relationship was found between the number of deformities and the administration of drugs.

We examined the relationship between the administration of drugs and the rate of change of BMD. The group who received no drugs, the group who received the calcitonin preparation alone, and the group who received a combination of active vitamin D3 and calcium preparations all showed an increase in BMD according to the DEXA results. The mean rates of change of BMD for these three groups were $0.005 \pm 0.028,0.0009 \pm 0.031$ and 0.006 \pm 0.031 , respectively. However, only two groups showed an increase in BMD according to the QCT results: the group who received active vitamin D3 alone and the group who received a combination of active vitamin D3 and calcium preparations. The mean rates of change of BMD for these two groups were $0.041 \pm 0.80$ and $0.036 \pm 0.171$, respectively. The group who received a combination of active vitamin D3 and calcium preparations was therefore the only group to show a positive change of BMD according to both the DEXA and QCT results. However, there was no significant difference among the groups. The group who received a combination of active vitamin D3 and calcium preparations was not compared with the group treated with a combined administration of active vitamin D3 and calcitonin preparations, because the former group consisted of only seven patients whereas the latter comprised 47 patients.

We also examined the relationship between the administration of drugs and changes in vertebral deformity. There was no significant difference in

TABLE 2.

$B M D$ was not related to progression of an existing deformity or development of an additional deformity

\begin{tabular}{lccc}
\hline & Number & Rate of change DEXA & Rate of change QCT \\
\hline Progress (trauma) & 6 & $-0.0089 \pm 0.048 \square @ 0.048$ & $-12.14 \pm 26.01 \square @$ \\
Another vertebra (no trauma) & 6 & $-0.0378 \pm 0.048$ & $-0.979 \pm 0.853$ \\
\hline
\end{tabular}

@ N.S 
64 year old woman BMD $0.351 \mathrm{~g} / \mathrm{cm}^{2}$

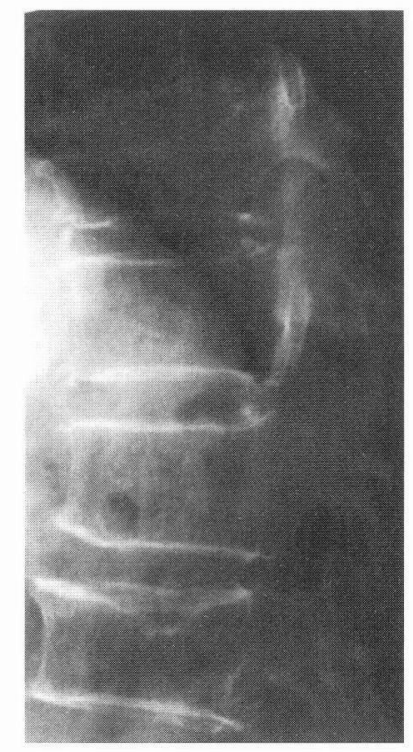

First examination
13 years post menopausal

BMD 0.343g/ $\mathrm{cm}^{2}$

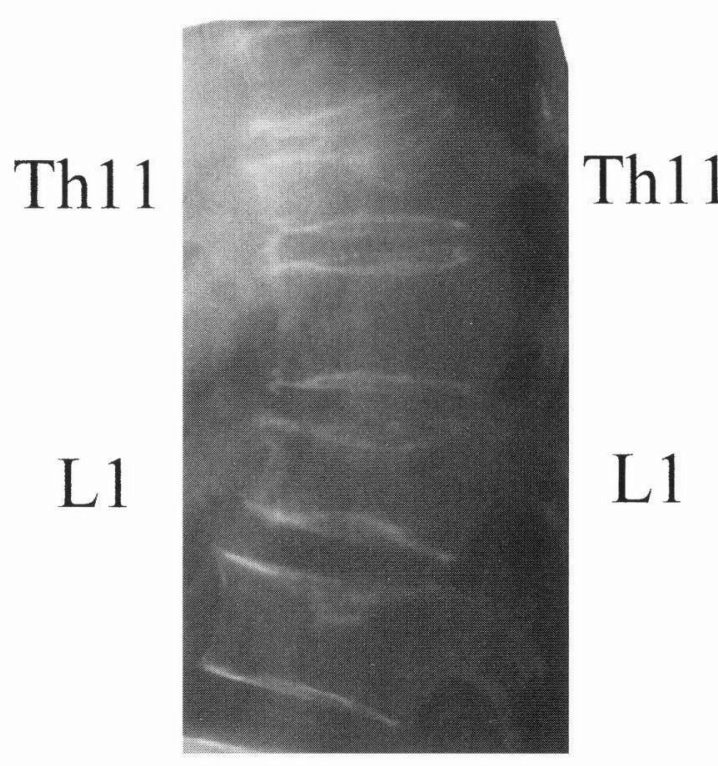

After one year

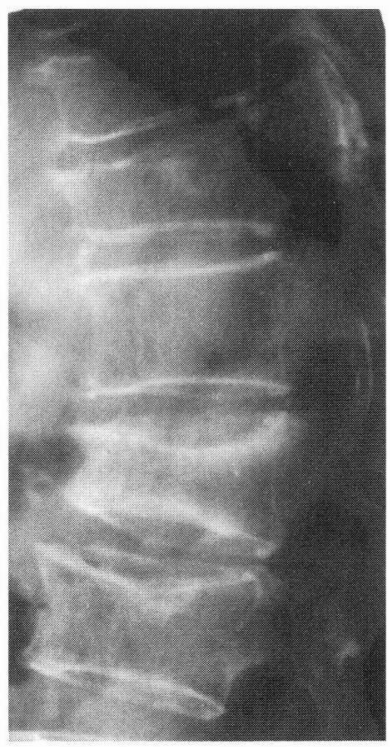

After 13 months

Fig. 7. There was no change in the Th1 1 compression fracture but a new fracture occurred at L1 during the followup period.

changes in vertebral deformity between the groups. Therefore, no combination of the drugs tested was able to prevent the progression of vertebral deformities.

\section{Case study}

This case involved a 64-year-old woman (13years postmenopausal) who had suffered from back pain with no known cause for 1 week. An acute 11th thoracic vertebra compression fracture was identified on a plain radiograph during the initial medical examination. We initiated treatment for osteoporosis consisting of a combination of intramuscular injections of calcitonin and an active vitamin D3 preparation. Although the collapsed 11th thoracic vertebra showed no progression after 1 year, plain radiography revealed a new compression fracture in the 1st lumbar vertebra, again with no known cause. The back pain had ceased about 2 months after the initial medical examination and the patient was able to carry out normal daily activities without difficulty. One month after the 12-month follow-up examination, the collapsed first lumbar vertebra had progressed, although the back pain had not increased. BMD (DEXA) was $0.351 \mathrm{~g} / \mathrm{cm}^{2}$ at the initial medical examination and had decreased to $0.343 \mathrm{~g} / \mathrm{cm}^{2}$ after 1 year: the rate of change of BMD was $2.3 \%$ per year (Fig. 7).

\section{DISCUSSION}

In cases of osteoporosis, the symptoms indicating vertebral fracture generally improve over a period of several weeks or months without complicating paralysis in the patient. Recently, however, late paralysis after vertebral compression fractures has been reported in osteoporotic spine disorders. The number of vertebral body deformities increases with age and patients often suffer disturbance to their activities of daily living (ADL) as a result of kyphotic deformity or chronic pain.

It is commonly thought that the lower the BMD the higher the risk of occurrence of vertebral deformities (vertebral fractures). However, among individuals with similar BMD, some develop deformities and others do not. As a result, methods for setting fracture thresholds vary between studies. For example, Suzuki et al. [1] defined the fracture threshold, measured using QCT, as $\leq 50 \%$ of the value in healthy individuals of the same age group, whereas 
Ono et al. [2] considered $\leq 100 \mathrm{mg} / \mathrm{cm}^{3}$ to be the threshold. In addition, in order to include $90 \%$ of all compression fractures, Riggs et al. [3] calculated the threshold as $0.965 \mathrm{~g} / \mathrm{cm}^{2}$ measured using dual photon absorptiometry (DPA), Habu et al. [4] gave a value of $0.59 \mathrm{~g} / \mathrm{cm}^{2}$ ( $72 \%$ of the maximum bone mass) and Ryan et al. [5] defined the threshold as $0.81 \mathrm{~g} / \mathrm{cm}^{2}$ measured using DEXA.

These previous reports have considered atraumatic vertebral body deformities, and the relationship between trauma and deformity has not been clearly defined. Medical examinations of outpatients often reveal that fractures have been sustained as a result of slight trauma. We therefore investigated the BMD of patients with osteoporosis aged $\geq 50$ years, including those who had a history of slight trauma (excluding high-energy traumas such as road traffic accidents and falls from a significant height).

Our cross-sectional study revealed significant differences in mean BMD among the different age groups in our study population: the older the age group the lower the BMD, according to both the DEXA and QCT results. This trend is consistent with that reported in previous studies. In addition, we found that the older the age group the greater the number of vertebral body deformities, which is also consistent with previous reports.

In terms of the relationship between age and the type of deformity present, the mean age of patients with fish vertebral deformities was the highest, followed by those with flat vertebrae, irregular endplates, wedged vertebrae and no deformities, in that order. The fish vertebral deformity differed from the other types in that no spondylosis deformans was observed in most of the vertebrae. In addition, there was no sclerosis with bone spur formation in the true fish vertebral deformity and the continuity of the endplates was maintained, although they were thin. A total of $60 \%$ of the patients with fish vertebrae had an atraumatic deformity without bone spur formation, whereas the remaining $40 \%$, who had suffered some kind of trauma, showed spondylosis deformans. In each of the other types of deformity, approximately one-half of the cases were associated with trauma: $50 \%$ of those with irregular endplates, $50.3 \%$ of those with wedged vertebrae and $40 \%$ of those with flat vertebrae. The true fish vertebral deformity, unlike the other types, showed no break in the endplate and appeared to be the result of a decrease of BMD in the cancellous bone when the internal pressure of the intervertebral disc was maintained to some degree. The other types of deformity appeared to generally start with an irregularity of the endplate, which is a coating on the vertebral body, as a result of a trauma that the patients themselves might not be aware of. These irregularities then progressed through the wedged and flat vertebral stages.

In atraumatic cases, patients with no vertebral deformity showed a significantly higher mean BMD (DEXA $=0.395 \pm 0.06 \mathrm{~g} / \mathrm{cm}^{2} ; \quad \mathrm{QCT}=80.5 \pm 38.2 \mathrm{mg} /$ $\mathrm{cm}^{3}$ ) compared with those with single or multiple vertebral body deformities, which was consistent with previous reports. By contrast, the groups that were associated with some kind of trauma showed a slightly lower BMD, according to the number of vertebral body deformities, compared with the atraumatic group. However, there was no statistically significant difference between the two groups. Among those with a BMD of around $0.42 \mathrm{~g} / \mathrm{cm}^{2}$ (DEXA) or $90 \mathrm{mg} / \mathrm{cm}^{3}$ (QCT), some patients had sustained deformities as a result of slight trauma in their daily lives, such as vomiting or performing load-lifting tasks. However, even among those with a BMD $>0.45 \mathrm{~g} / \mathrm{cm}^{2}$ (DEXA) or $120 \mathrm{mg} / \mathrm{cm}^{3}$ (QCT), there were some patients who showed deformities. The BMD levels that included $90 \%$ of all patients with deformities were $0.424 \mathrm{~g} / \mathrm{cm}^{2}$ (DEXA) and 94.86 $\mathrm{mg} / \mathrm{cm}^{3}$ (QCT), which seemed to represent the threshold above which fractures owing to trauma did not occur.

In our longitudinal study, we examined 105 patients after a six-month follow-up period. A total of $82 \%$ of these patients had either no deformity or no progression of existing deformities after six months; however, the remaining $18 \%$ were clinically significant. A total of $40 \%$ of the patients who showed progression of an existing deformity and $87.5 \%$ of the patients who developed an additional vertebral deformity during the follow-up period had not reported trauma at the initial medical examination. Although there was no significant difference between the groups, those who developed an additional vertebral deformity during the follow-up period therefore tended not to have reported trauma at the initial medical examination. There was no significant difference in the mean BMD recorded at the initial medical examination for these groups; therefore, initial BMD was not associated with the observed changes in deformity.

Patients with existing deformities that progressed during the follow-up period included those who had irregular endplates, wedged vertebrae and flat vertebrae at the initial medical examination. However, patients with initial fish vertebral deformities showed 
no progression during the follow-up period. Therefore, as in the cross-sectional study, fish vertebral deformities could be separated from other patterns of deformity. In addition, $58 \%$ of the group that showed no progression of existing deformities during the follow-up period had wedged vertebral deformities at the initial medical examination. Therefore, wedged vertebrae showed a tendency not to progress.

We examined the relationship between the rate of change of BMD and the number of vertebral body deformities present at the initial medical examination. There was no significant difference between the rate of change in the groups with no deformities, single vertebral body deformities and multiple vertebral body deformities. Therefore, there was no evidence that increased numbers of vertebral body deformities were associated with a greater rate of decrease in BMD. In addition, there was no significant difference in the rate of change of BMD among any of the groups, according to both the DEXA and QCT results. We therefore concluded that BMD was not associated with the progression of deformities or changes in the pattern of deformities.

We also investigated the relationship between the rate of change of BMD and the existence of deformities. The subjects were classified into two groups: one containing patients who showed no deformities during the follow-up period and the other containing all those who showed any type of deformation. There was no significant difference between these two groups in the rate of change of BMD. We then examined the relationship between the rate of change of BMD and the existence of change within deformity patterns. Again, the subjects were classified into two groups: one consisting of those who had no deformity and those who showed no progression of an existing deformity (the group with no change), and the other consisting of all those who exhibited any change during the observation period (the group with change). Although there was no significant difference between the two groups according to the DEXA results, there was a significant difference in the QCT results at the $5 \%$ probability level $(\mathrm{p}<$ 0.005 ). We therefore concluded that decreased BMD was one possible cause of the progression of deformities. Among the group with change, 17 of the 19 patients showed a progression in existing deformities without any obvious cause during the follow-up period. It therefore seemed likely that daily occurrences, of which the patients were not aware, could have been related to the progression of deformities.
In our analysis of the influence of drug administration on changes in BMD during the follow-up period, the group who received a combination of both active vitamin D3 and calcium preparations showed a positive rate of change of BMD according to both the DEXA and QCT results. However, because there were no significant differences among the groups receiving different drug treatments, none of the treatments investigated caused a significant increase in BMD. However, in terms of the maintenance of BMD levels, the combined vitamin D3 and calcium preparations seemed to be effective.

No significant difference was seen in the change of deformity patterns according to the administration of drugs among the study groups. It therefore appeared that none of the drugs investigated could prevent the progression of deformities.

\section{CONCLUSION}

Many researchers have reported that decreased BMD seems to cause vertebral body deformities. However, we found it difficult to predict whether deformities will progress over time or lead to the development of additional deformities of the adjacent vertebral bodies. This is because the major contributing factors to the development and progression of vertebral body deformities include not only BMD and age but also lifestyle and activity patterns. In addition, none of the methods of drug administration investigated here resulted in a notable increase in BMD.

\section{REFERENCES}

1. Suzuki S, Okumura H, and Yamamuro T. Trabecular mineral contents of lumbar vertebra in patients with osteoporosis. J Jpn Orthop Assoc 1990; 64:17-26. (in Japanese)

2. Ono K, Susuda K, and Higuchi M. Examination of relationship between thoraco- lumbar vertebra compression fracture and bone mineral density in women by QCT and DEXA. Jpn Bone Metab Assoc 1990; 8:280. (in Japanese)

3. Riggs BL, Wahner HW, and Dunn WL. Differential changes in bone mineral density of the appendicular and axial skeleton with aging. J Clin Invest 1981; 67:328335.

4. Habu T, and Takahashi E. Relationship between decrease of bone mineral density and fracture in aged people. Orthop Mook 1991; 62:10-17. (in Japanese)

5. Ryan PJ, Blake GM, and Fogelman I. Fracture thresholds in osteoporosis, implications for hormone replacement treatment. Anna Rheum Disease 1992; 51:1063-1065. 